\title{
Title:
}

\section{What are the odds? Duodenum adenocarcinoma as a primary metachronic neoplasia}

\section{Authors:}

Asunción Ojeda Gómez, Almudena Íñigo Chaves, Lucía Madero Velázquez, Jorge Barragán Martínez, Maria Dolores Picó Sala, Andrea García Soria, Julia Sáez Fuster, Mariana Fe García Sepulcre

DOI: $10.17235 /$ reed.2021.8200/2021

Link: PubMed (Epub ahead of print)

Please cite this article as:

Ojeda Gómez Asunción, Íñigo Chaves Almudena, Madero Velázquez Lucía, Barragán Martínez Jorge, Picó Sala Maria Dolores, García Soria Andrea, Sáez Fuster Julia, García Sepulcre Mariana Fe. What are the odds? Duodenum adenocarcinoma as a primary metachronic neoplasia. Rev Esp Enferm Dig 2021. doi: 10.17235/reed.2021.8200/2021.

This is a PDF file of an unedited manuscript that has been accepted for publication. As a service to our customers we are providing this early version of the manuscript. The manuscript will undergo copyediting, typesetting, and review of the resulting proof before it is published in its final form. Please note that during the production process errors may be discovered which could affect the content, and all legal disclaimers that apply to the journal pertain. 


\section{What are the odds? Duodenum adenocarcinoma as a primary metachronic neoplasia}

Asunción Ojeda Gómez, Almudena María Înigo Chaves, Jorge Barragán Martínez, Lucía Madero Velázquez, María Dolores Picó Sala, Andrea García Soria, Julia Sáez Fuster, Mariana Fe García Sepulcre

Gastroenterology Service. Hospital General Universitario de Elche.

No conflicts of interest.

\section{Correspondence:}

Asunción Ojeda Gómez

Email: asunojedagomez@gmail.com

We present a 81-year-old man with a history of colorectal and bladder cancer surgically removed, who was admitted to our hospital with a urinary infection. A routine renal ultrasound revealed a pelvic cyst and CT-scan confirmed a noncomplicated cyst but observed a lesion in the left lung. He underwent a PET-CT in which an unexpected enhancement of the small bowel called our attention. The patient had always been asymptomatic from a gastrointestinal point of view and his blood work was unremarkable. An oesophagogastroduodenoscopy was carried out, founding a lonely large mass in the third portion of the duodenum that turn out to be a primary duodenal adenocarcinoma (PDA). The histopathological analysis revealed Marsh 0 and no genetic disorder was found. The committee proposed him for a segmentary resection without neoadjuvant treatment, and a later semestral follow-up with image tests. Nevertheless, the patient died because of a surgical complication.

PDA accounts for $0.3-1 \%$ of all gastrointestinal cancers. While most tumors of the ileum are neuroendocrin, adenocarcinoma is the most common duodenal cancer, arising almost always in D2. A recognised risk factor is PAF syndrome (1) (2). With an 
asymptomatic or nonspecific presentation such as abdominal discomfort or occult bleeding, patients are usually misdiagnosed (3). An oesophagogastroduodenoscopy with biopsies remains the first-line diagnostic procedure, but an enteroscopy or capsule endoscopy are needed for lesions beyond D2 (4).Duodenal adenomas should always be endoscopically removed (5). However, when worrying features are noted, such as size greater than $20 \mathrm{~mm}$, Paris IIc-III, presence of ulcerations or loss of the pit pattern, biopsies should be taken. Tumors in D2 typically require pancreaticoduodenectomy whereas other locations could be managed with segmentary resections. A wide lymphadenectomy should always be performed, since lymph node positivity is the most important prognosis indicator. With regards to the adjuvant therapy and the follow-up, there is no established protocol due to lack of randomized controlled trials.

Keywords: Primary adenocarcinoma. Duodenum. Endoscopy.

\section{References:}

1. Khanal S, Joshi U, Bhattarai A et al. Primary Duodenal Adenocarcinoma: Uncommon Tumor, Uncommon Presentation. A Case Report and Review of the Literature. Int Med Case Rep J. 2020

2. May 15;13:165-169. doi: 10.2147/IMCRJ.S256107. PMID: 32523384; PMCID: PMC7237129.

Ocaña J, López A Nuño J. Poliposis adenomatosa familiar: manejo quirúrgico. Duedenectoía con preservación pancreática o cirugía de Whipple. Rev Esp Enferm Dig. 2019;11:572-573

3. You HS, Hong JW, Yun EY et al. Primary Non-ampullary Duodenal Adenocarcinoma: A Single-center Experience for 15 Years. Korean J Gastroenterol. 2015 Oct;66(4):194-201. Korean. doi: 10.4166/kjg.2015.66.4.194. PMID: 26493504.

4. Paquissi F, Lima A, Lopes $F$ et al. Adenocarcinoma of the third and fourth 
portions of the duodenum: The capsule endoscopy value. World J Gastroenterol. 2015 Aug 21;21(31):9437-41. doi: 10.3748/wjg.v21.i31.9437. PMID: 26309371; PMCID: PMC4541397.

5. Vanbiervliet. Endoscopic management of superficial nonampullary duodenal tumors: European Society of Gastrointestinal Endoscopy (ESGE) Guideline. Endoscopy 2021; 53: 522-534.

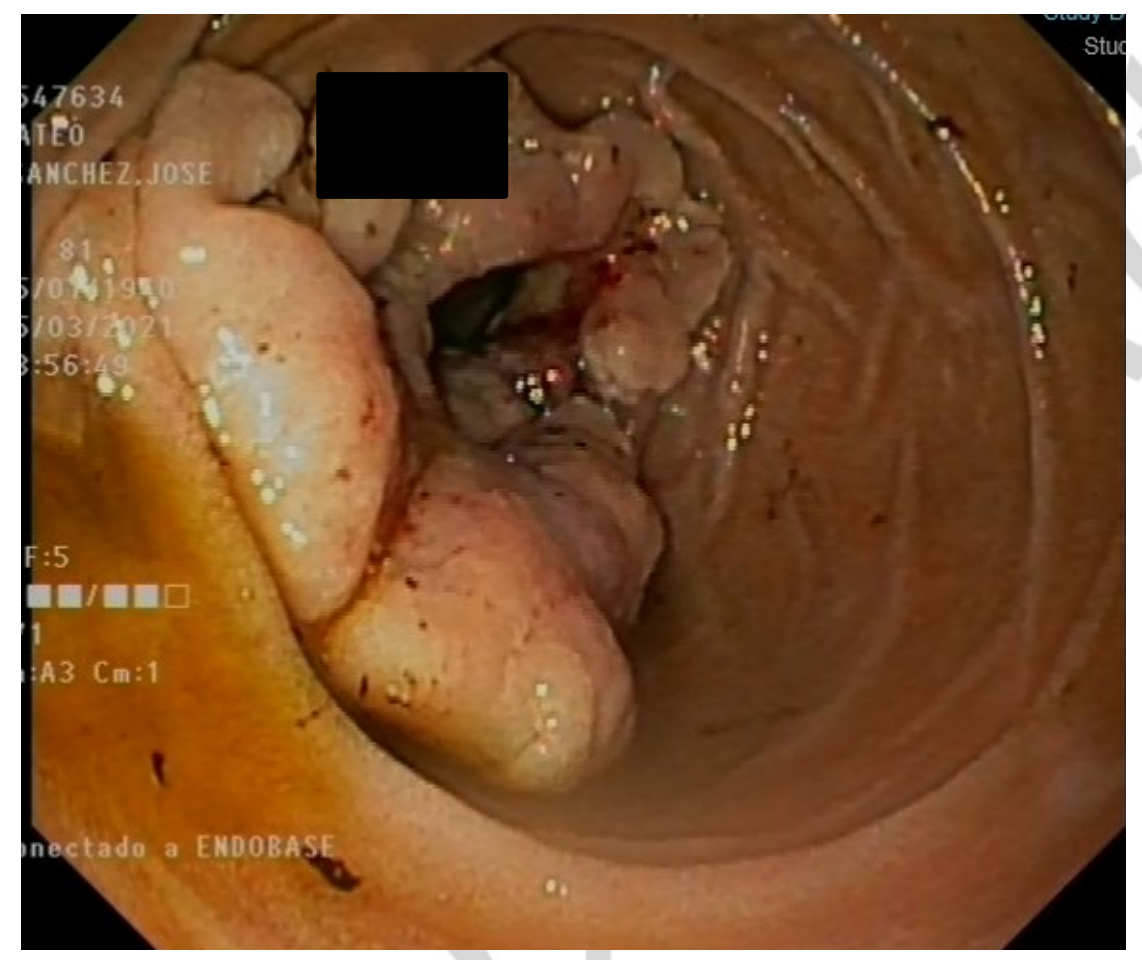

Image 1. We observe a $30-40 \mathrm{~mm}$ mass in the third part of the duodenum, occupying $50 \%$ of the lumen but easily passable with the scope, with a large ulceration with fibrin base and without any signs of recent bleeding. 


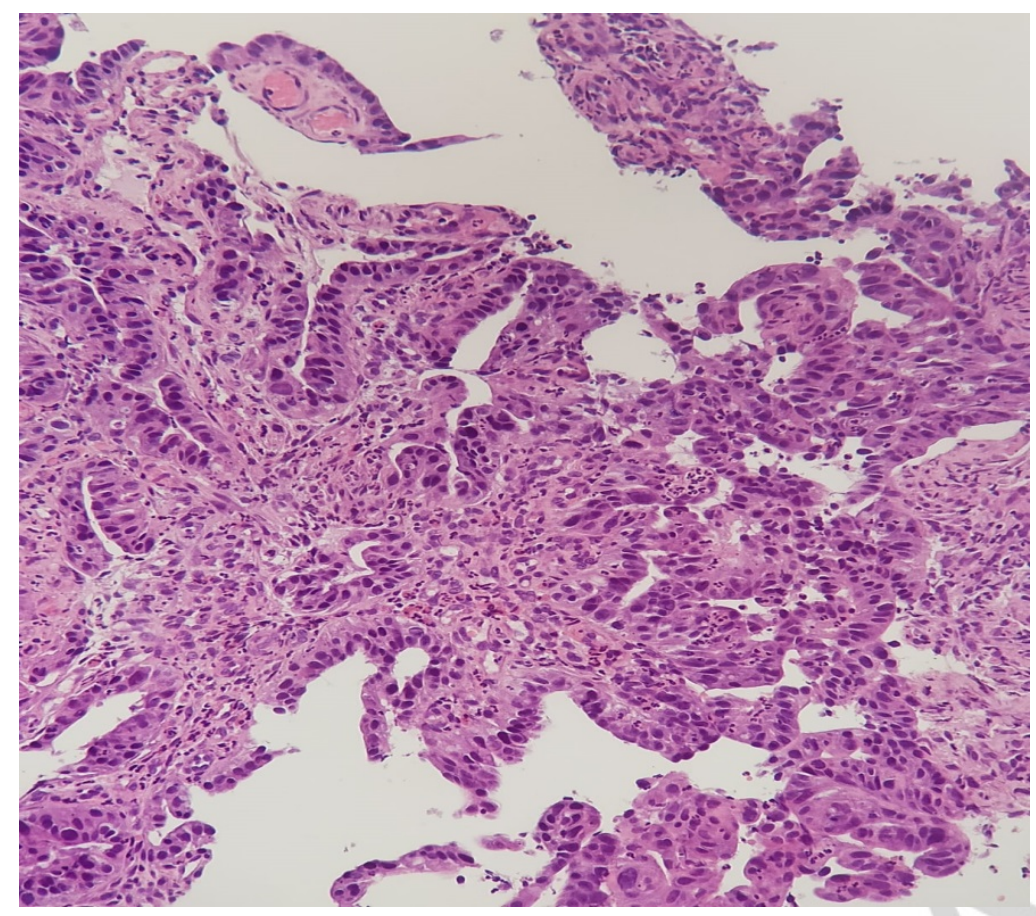

Image 2. Primary duodenum adenocarcinoma 